\title{
Modeling of a Fuel Tank with an Air Compressor
}

\author{
Çağrı Odabaş $1^{*}$ and Mesut Düzgün ${ }^{1}$
}

${ }^{1}$ Automotive Engineering Department, Faculty of Technology, Gazi University, Ankara, 06500, Turkey

\begin{abstract}
It was observed that approximately $10 \%$ of the fuel remains trapped in the fuel tank unable to be used during operation of cars on fields with an elevation difference of up to $25^{\circ}$ in $\mathrm{x}$ and y directions. To overcome this problem, a new type of fuel tank is designed which is based on the idea of implementing a movable partition in the fuel tank that will work to push the fuel within the tank towards the suction pump as the fuel in the tank drained through car use. In this paper, the mathematical model of the fuel tank was found and the transfer function was given in terms of the amount of fluid inside the tank and the displacement of the partition. In addition, from the transfer function, the pole-zero map of the system and the root-locus were drawn. Finally, a PID controller was designed for this fuel tank.
\end{abstract}

Keywords: Air compressor; Fuel tank; Implementing partition; Modeling; PID controller; Pole-zero map; Root-locus; Transfer function
* Corresponding author

Çağrı Odabaşı

cagriodabasi94@gmail.com

Adress: Automotive Engineering Department, Faculty of Technology, Gazi University, Ankara, Turkey

Phone:+903122846922

Manuscript Received 01.02.2018

Revised 21.04.2018

Accepted 25.05.2018

Doi:10.30939/ijastech..388631

\section{Introduction}

During operation of cars on fields with an elevation difference of up to $25^{\circ}$ in $\mathrm{x}$ and $\mathrm{y}$ directions showed in Figure 1 it was observed that approximately $10 \%$ of the fuel remains trapped in the fuel tank unable to be used. This happens because the suction system used in the fuel tank is unable to reach the fuel that remains in the tank when the tank is nearly empty during car operations under elevated conditions for long periods of time. To overcome this problem, a new type of fuel tank is designed which is based on the idea of implementing a movable partition in the fuel tank that will work to push the fuel within the tank towards the suction pump as the fuel in the tank is drained through car use. For this concept, it is inspired from the "Fuel Storage Apparatus for Vehicle" patent. ${ }^{[1]}$ The concept will function by taking advantage of the pressure in the fuel tank. When the tank is full, the partition will remain at the $x=0$ location in the fuel tank. As the car operates, the suction system will supply fuel to the engine, which will consume the fuel in the fuel tank and thus drain it. As the fuel tank starts to drain, the pressure in the tank will start to decrease which will result in the partition to start moving from the $\mathrm{x}=0$ location to the location of the suction system. As the partition moves, it will dislocate the remaining fuel in the tank so that it gets closer to the suction system. As a result of this, the height of the fluid in the tank will remain constant. This will allow the suction system to always supply the car with the fuel in the fuel tank. Any and all elevations will not matter as the moving partition will ensure that the fuel in the tank continually moves towards the suction system to be used.

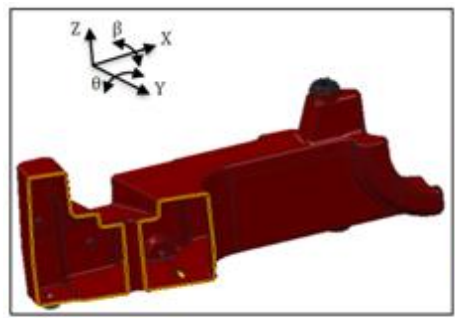

(a)

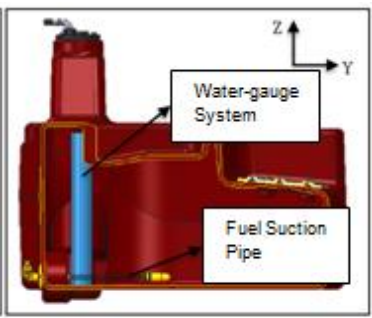

(b)
Fig. 1. Different cut planes of the fuel tank 
In order for this system to function, a source of pressure that will enable the partition to move is required. This pressure should be nearly equal to but less than the pressure of the fuel tank when the tank is full. The pressure in the fuel tank will decrease as the fuel in the tank is directed to the engine and consumed in accordance with the working principles of the fuel tank. Since the pressure that will move the partition is nearly equal to but still less than the full fuel tank pressure, the partition will start moving once the pressure in the fuel tank decreases past a certain threshold value in the fuel tank as the tractor operates. This will enable the partition to move from the $\mathrm{x}=0$ location to the suction system, allowing the fuel within the tank to be redirected to the suction system regardless of the percentage of fuel remaining in the tank or the location of the fuel as a result of the elevation angle. This pressure can be supplied to the tank by using an air compressor to supply pressurized air into the partition. The pressurized air that is supplied to the tank should be less than but nearly equal to the pressure of the fuel tank when the tank is full for the reasons discussed above. A rough drawing of the design of this system is supplied as Figure 2 below.

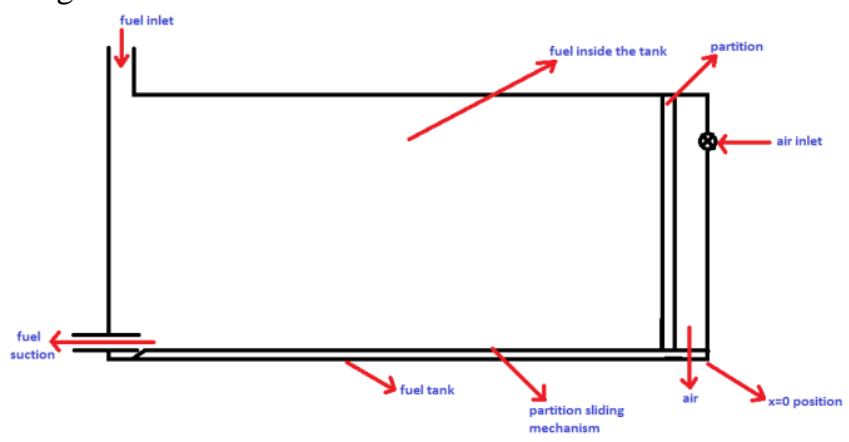

Fig. 2. The concept as applied to the fuel tank

Before the operation begins, the fuel tank is filled with fuel and the partition is at the $x=0$ location. In this situation, the air compressor is still functional and is giving the pressurized air from the outside into the partition. However, since the pressure of the pressurized air that is pumped inside the air is less than the pressure of the full fuel tank, the partition will not move. As the system operates, the pressure of the fuel tank will decrease as elaborated above, as a result of this decrease, the partition will start to move as the pressure of the compressed air is nearly equal to the pressure of the tank at full. In this way, the concept depicted will function from the time that the fuel tank is full to the time that the fuel tank is nearly empty. Thus, this concept allows for the use of nearly all of the fuel in the tank.

The actual fuel tank has a far more irregular shape but for Figure 2, it is assumed that the fuel tank is of a general shape as rectangle. Since the geometry of the fuel tank is irregular, the implementation of this partition is very difficult. The partition needs to be implemented in segments along the irregular shape of the length of the tank. As the tank drains and the partition starts sliding towards the suction system, the segments of the partition seal and start to slide together until the partition reaches the suction system. However, for the calculations, the tank geometry is assumed as like Figure 2 which has length $L$, width $w$ and height $h$.

\section{Justification}

With current fuel tanks, when car stops itself because of the lack of the fuel, there will be remaining fuel inside the tank under elevated conditions. This causes a problem in terms of cost efficiency. For the main motivation to solve this problem, before selling process of the cars, companies need to provide some fuel inside the tank because customers need to be able to go to the nearest gas station or their home by car. The companies want to fill the tank with minimum required fuel in order to get maximum profit; however, the existing system does not operate with less than $10 \%$ fuel in $25^{\circ}$ working angle. Hence, they need to fill at least $15 \%$ for all the cars they are selling in order to make the car arrive the nearest fuel station. This creates considerable cost when considering the number of products they sell.

In the right-hand side of the partition, there is a gas which can be supplied by the internal combustion engine and controls the movement of the partition. In an internal combustion engine, the expansion of the hightemperature and high-pressure gases produced by combustion apply direct force to the components of the engine and the resultant pressurized gases can be transmitted to move the partition when there is a pressure difference between left-hand side and right-hand side. As a result of the pressure on the left-hand side which applies force to the partition, it is expected to have a constant fuel height in the fuel tank.

\section{Calculations ${ }^{[2]}$}

For this project, an incompressible flow is considered which is an idealized case, wherein the variation of pressure, temperature, etc., encountered by a particle cause zero change in the density of the particle. The variables of the fluid flow systems are pressure, fluid flow rate and volumetric flow rate where $\dot{m}=\rho . Q$.

The useful formulas for the calculations of the fluid flow system are given below.

$$
\begin{aligned}
& \Delta p=p_{2}-p_{1}=I \cdot \frac{d Q}{d t} \\
& Q=C_{f} \cdot \frac{d(\Delta p)}{d t} \\
& \Delta p=p_{2}-p_{1}=Q \cdot R
\end{aligned}
$$

Eq. (3) is valid for laminar flows.

$Q=\frac{1}{R} \cdot\left(p_{2}-p_{1}\right)^{1 / \alpha}$

In Eq. (4), $\alpha$ is 2 for high flow rates.

From conservation of mass for incompressible fluids and closed conduits, 
$\dot{m}=W_{\text {in }}(t)-W_{\text {out }}(t)$

For the partition, from the force balance, $F=p . A$. When using a partition with a mass in dynamic analysis, Newton's second law can be applied,

$$
\text { p.A-Fr }=m_{p} \cdot \ddot{x}
$$

Without considering the partition, first it is needed to find $W_{\text {out }}(t)$. In order to find it, resistive element relationship (Eq. (3)) is used with assuming laminar flow. Since the pressure difference is $\rho . g . h$,

$$
Q_{\text {out }}(t)=\frac{1}{R} \cdot \rho \cdot g \cdot h(t)
$$

With combining Eq. (7) and $\dot{m}$ expression,

$$
W_{\text {out }}(t)=\frac{\rho^{2} * g}{R} \cdot h(t)
$$

Substituting Eq. (8) in the Eq. (5) gives,

$$
\dot{m}=W_{i n}(t)-\frac{\rho^{2} \cdot g}{R} \cdot h(t)
$$

With taking Laplace of the Eq. (9),

$$
s \cdot M_{f}(s)-m_{f}(t=0)=W_{i n}(s)-\frac{\rho^{2} \cdot g}{R} \cdot H(s)
$$

Before calculating $m(t)$, it is necessary to replace for height in terms of mass of the liquid inside the tank. By observation,

$$
m_{f}(t)=A \cdot h(t) \cdot \rho
$$

The surface area of the tank,

$$
A=x(t) . w
$$

With combining Eq. (11) and Eq. (12) and taking Laplace of the resultant equation,

$$
M_{f}(s)=X(s) \cdot w \cdot H(s) \cdot \rho
$$

Then, substitute $H(s)$ in the Eq. (13) into Eq. (10).

$$
s \cdot M_{f}(s)-m_{f}(t=0)=W_{i n}(s)-\frac{\rho^{2} \cdot g}{R} \cdot \frac{M_{f}(s)}{X(s) \cdot w \cdot \rho}
$$

It was assumed that tank is full with fuel initially $(\mathrm{x}=0$ and $\left.m_{f}(t=0)=\rho \cdot x_{0} \cdot w \cdot h_{0}\right)$ and starts operating now.

With collecting all $M_{f}(s)$ terms in one side,

$$
M_{f}(s)=\frac{\rho \cdot x_{0} \cdot w \cdot h_{0}}{s+((\rho \cdot g) /(R \cdot X(s) \cdot w))}
$$

Rearranging the Eq. (15),

$$
X(s)=\frac{\rho \cdot g \cdot M_{f}(s)}{R \cdot w \cdot\left(\rho \cdot x_{0} \cdot w \cdot h_{0}-s \cdot M_{f}(s)\right)}=\frac{g}{R \cdot w^{2} \cdot x_{0} \cdot h_{0}} \cdot M_{f}(s)-\frac{1}{R \cdot w \cdot s}
$$

With taking inverse Laplace of Eq. (16),

$$
x(t)=\frac{g}{R \cdot w^{2} \cdot x_{0} \cdot h_{0}} \cdot m_{f}(t)-\frac{1}{R \cdot w}
$$

However, there is an error in the Eq. (17) since when there is no fuel in the tank $\left(m_{f}(t)=0\right)$, according to this equation $x(t)$ should be negative which cannot be possible. Therefore, it is decided to use Eq. (6). Since pressure applied by fuel to the midpoint of the partition is $\rho . g \cdot \frac{h(t)}{2}$, Eq. (6) becomes

$$
\rho \cdot g \cdot \frac{h(t)}{2}-F_{r}=m_{p} \cdot \ddot{x}(t)
$$

From Eq. (11) and (12),

$$
m_{f}(t)=x(t) \cdot w \cdot h(t) \cdot \rho
$$

With substituting Eq. (19) into Eq. (18),

$$
\ddot{x}(t)=\frac{g}{2 \cdot w \cdot m_{p}} \cdot \frac{m_{f}(t)}{x(t)}-\frac{F_{r}}{m_{p}}=F\left(x(t), m_{f}(t)\right)
$$

Since Eq. (21) is non-linear, it should be linearized. The partition is operating mostly around at $x=L / 2$ and corresponding fuel mass is $m_{f}=M / 2$, so the Eq.(20) is linearized around these points. Resultant linarized equation is in the form of,

$$
\ddot{x}(t)+a_{1} \cdot \dot{x}(t)+a_{0} \cdot x(t)=b_{1} \cdot \dot{m}_{f}(t)+b_{0} \cdot m_{f}(t)^{[3]}
$$

where,

$$
\begin{aligned}
& a_{1}=-\frac{\partial F}{\partial \dot{x}} \\
& a_{0}=-\frac{\partial F}{\partial x} \\
& b_{1}=\frac{\partial F}{\partial m_{f}} \\
& b_{0}=\frac{\partial F}{\partial m_{f}}
\end{aligned}
$$

When $a_{1}, a_{0}, b_{1}$ and $b_{0}$ are calculated according to Eq. (22), (23), (24) and (25) respectively and substituting 
$x=L / 2$ and $m_{f}=M / 2$ and with substituting the results in Eq. (21),

$$
\ddot{x}(t)+\frac{g \cdot M}{w \cdot m_{p} \cdot L^{2}} \cdot x(t)=\frac{g}{w \cdot m_{p} \cdot L} \cdot m_{f}(t)
$$

Eq. (26) is the linearized version of Eq. (20) around $x=L / 2$. Since Eq. (26) is linear now; it can be expressed in Laplace domain.

$$
\left(s^{2}+\frac{g \cdot M}{w \cdot m_{p} \cdot L^{2}}\right) \cdot X(s)=\frac{g}{w \cdot m_{p} \cdot L} \cdot M_{f}(s)
$$

Hence, system's transfer function is,

$$
\frac{M_{f}(s)}{X(s)}=\frac{s^{2}+\frac{g \cdot M}{w \cdot m_{p} \cdot L^{2}}}{\frac{g}{w \cdot m_{p} \cdot L}}
$$

From Eq. (28), the relation between mass of fuel and the position of the partition is found as,

$$
m_{f}(t)=\frac{w \cdot m_{p} \cdot L}{g} \cdot \ddot{\mathrm{x}}(\mathrm{t})+\frac{\mathrm{M}}{\mathrm{L}} \cdot \mathrm{x}(\mathrm{t})
$$

\section{Pole-Zero Map}

From Eq. (28), it can be understood that there is no pole and the system has two zeros which are,

$$
\begin{aligned}
& z_{1}=\frac{1}{L} \cdot \sqrt{\frac{g \cdot M}{w \cdot m_{p}}} j \\
& z_{2}=-\frac{1}{L} \cdot \sqrt{\frac{g \cdot M}{w \cdot m_{p}}} j
\end{aligned}
$$

In order to sketch the pole-zero map, the parameters should be defined. In order to define the parameters fuel tank model given as Figure 3 is used which is a 25 liter fuel tank.

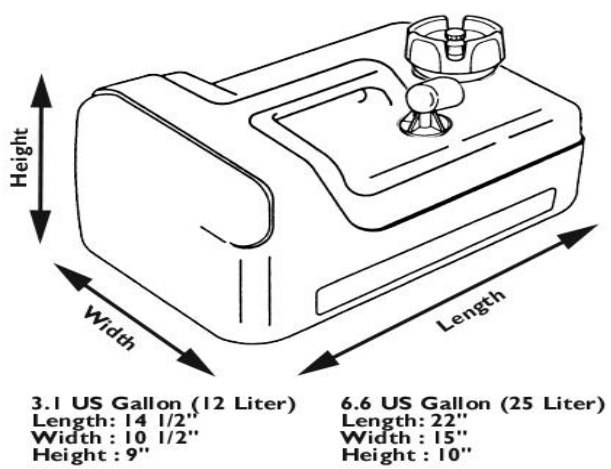

Fig. 3. The reference fuel tank ${ }^{[4]}$

$\mathrm{g}=10 \mathrm{~m} / \mathrm{s}^{2}$

$\mathrm{M}=20.8 \mathrm{~kg}$ (since the density of the petroleum diesel is $0.832 \mathrm{~kg} / \mathrm{L}$ )

$\mathrm{w}=38.1 \mathrm{~cm}$ $m_{p}=2 \mathrm{~kg}$

$\mathrm{L}=55.88 \mathrm{~cm}$

Therefore, the results of $\frac{g \cdot M}{w \cdot m_{p} \cdot L^{2}}$ and $\frac{g}{w \cdot m_{p} \cdot L}$ are found as 0.087 and 0.235 respectively and the transfer function becomes as $\frac{M_{f}(s)}{X(s)}=\frac{s^{2}+0.087}{0.235}$. With using Matlab, the pole-zero map can be drawn for this system as shown in Figure 4.

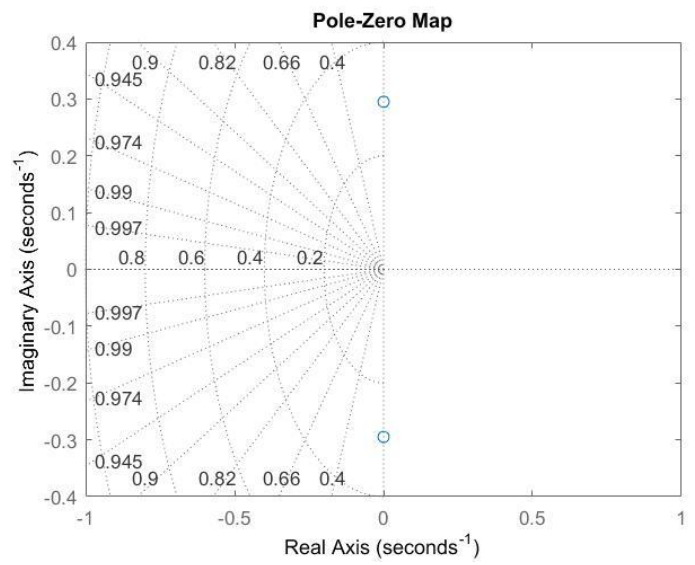

Fig. 4. Pole-zero map of the system

Since there is no pole, it can be said that the system is stable. Therefore, it is not necessary to look at RouthHurwitz stability criterion.

\section{Root-Locus}

In order to sketch root locus, the parameters and the results are used as defined in Pole-Zero Map part.

With using Matlab, the root locus can be drawn as follows,

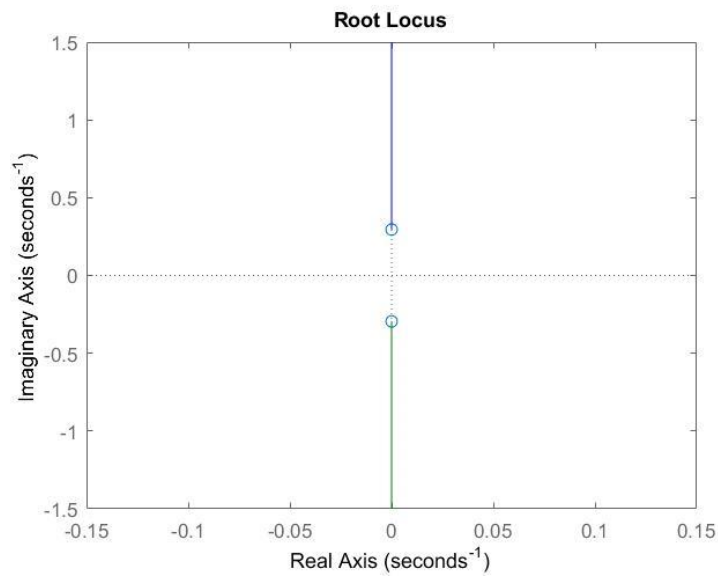

Fig. 5. Root locus of the system

\section{Designing a PID Controller}

Assuming that the unity feedback is considered, $H(s) \equiv 1$, system with the open loop transfer function becomes $G(s) H(s)=\frac{s^{2}+0.087}{0.235}$. Assume that it is desired to 
design a PID controller such that the unit step response of the above system has a maximum overshoot of $4.3 \%$ and a peak time of $0.5 \pi \mathrm{sec}$. Also, it is desired that the steady state error for unit step input to be zero and the dynamic error for ramp input to be $10 \%$.

The system step response performance specification imply the following requirements on the dominant closed loop pole locations $s_{1,2}$ and features of the open loop transfer function affecting the closed loop steady state characteristics:

- A maximum overshoot of $4.3 \%$, which means the damping ratio $\zeta=0.707$

- A peak time of $0.5 \pi$ sec., which means $w_{d}=2$ since $t_{p}=\frac{\pi}{w_{d}}=0.5 \pi$

Hence, $s_{1,2}=-2 \pm 2 j$

- Zero steady state error for unit step input, which means type number $=1$, so $K_{i} \neq 0$

- A dynamic error $10 \%$ for ramp input, which means $\frac{1}{K_{v}}=0.1$ and $\frac{30 . K_{i}}{40}=10$, so $K_{v}=10$ and $K_{i}=40 / 3=13.3$

In order to use the analytical method, the followings should be computed.

$$
s_{1}=\left|s_{1}\right| . e^{j \beta}
$$

where, $\left|s_{1}\right|=$

$2 \sqrt{2}=2.8284$ and $\beta=135^{\circ}=2.3562 \mathrm{rad}$

$$
G\left(s_{1}\right) \cdot H\left(s_{1}\right)=\left|G\left(s_{1}\right) \cdot H\left(s_{1}\right)\right| \cdot e^{j \varphi}
$$

where,

$$
\left|G\left(s_{1}\right) \cdot H\left(s_{1}\right)\right|=0.42903 \text { and } \varphi=138.2=
$$

\section{$2.4117 \mathrm{rad}$}

Using these values in the design equations give,

$$
\begin{aligned}
& K_{p}=\frac{-\sin (\beta+\varphi)}{\left|G\left(s_{1}\right) \cdot H\left(s_{1}\right)\right| \cdot \sin (\beta)}-\frac{2 \cdot K_{i} \cdot \cos \beta}{\left|s_{1}\right|} \\
&= \frac{-\sin \left(135^{\circ}+138 \cdot 2^{\circ}\right)}{(0.429) \cdot \sin \left(135^{\circ}\right)}-\frac{2 \cdot \frac{40}{3} \cdot \cos \left(135^{\circ}\right)}{2 \sqrt{2}}=9.5467 \\
& K_{d}=\frac{\sin (\varphi)}{\left|s_{1}\right| \cdot\left|G_{p}\left(s_{1}\right) \cdot H\left(s_{1}\right)\right| \cdot \sin (\beta)}+\frac{K_{i}}{\left|s_{1}\right|^{2}} \\
&=\frac{\sin \left(138 \cdot 2^{\circ}\right)}{(2 \sqrt{2}) \cdot(0.429) \cdot \sin \left(135^{\circ}\right)}+\frac{\frac{40}{3}}{(2 \sqrt{2})^{2}}=2.3467
\end{aligned}
$$

Hence, the transfer function of the PID controller becomes,

$$
\begin{aligned}
& G_{c}(s)=K_{p}+\frac{K_{i}}{s}+K_{d} s \\
& G_{c}(s)=9.35+\frac{13.3}{s}+2.35 s=\frac{2.35 s^{2}+9.35 s+13.3}{s}
\end{aligned}
$$

$$
G_{C}(s)=\frac{2.35\left(s^{2}+4.068 s+5.682\right)}{s}=\frac{13.3\left(1+0.716 s+0.176 s^{2}\right)}{s}
$$

The root locus of PID compensated system is given as Fig. 6.

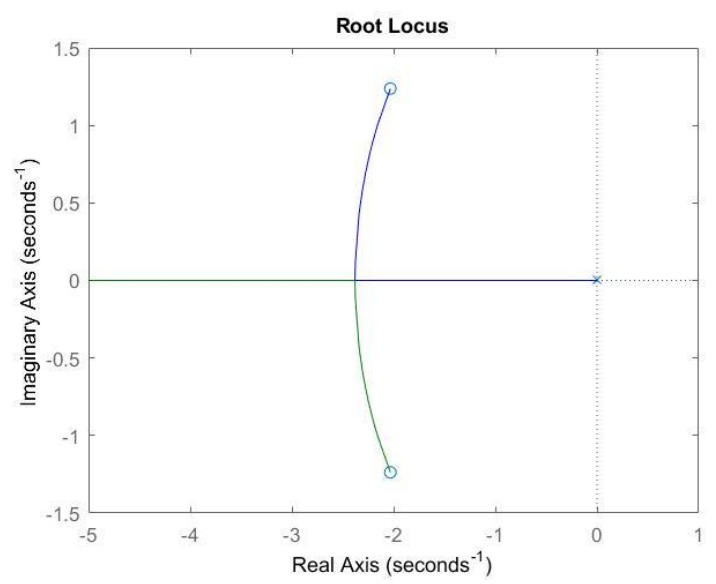

Fig. 6. The root locus of PID compensated system

Since $H(s) \equiv 1$ and $M(s)=\frac{G_{C}(s) G(s)}{1+G_{C}(s) G(s)}$, the closed loop transfer function of PID compensated system becomes,

$$
M(s)=\frac{G_{c}(s) G(s)}{1+G_{C}(s) G(s)}
$$

\section{Conclusion}

When a car operates with an elevation difference of up to $25^{\circ}$ in $\mathrm{x}$ and $\mathrm{y}$ directions, it was observed that approximately $10 \%$ of the fuel remains trapped in the fuel tank unable to be used because of the weakness of the fuel tank's suction system to reach the fuel that remains in the tank when the tank is nearly empty during car operations under elevated conditions for long periods of time. In this paper, to overcome this problem, a new type of fuel tank was designed which is based on the idea of implementing a movable partition in the fuel tank that will work to push the fuel within the tank towards the suction pump as the fuel in the tank is drained through car use. With this new fuel tank, when the car operates, the pressure of the fuel tank will decrease as elaborated above, as 
a result of this decrease, the partition will start to move as the pressure of the compressed air is nearly equal to the pressure of the tank at full. In this way, the concept depicted will function from the time that the fuel tank is full to the time that the fuel tank is nearly empty. Thus, this concept allows for the use of nearly all of the fuel in the tank. This paper mentions the exhaust of the internal combustion engine can be used for the air inlet where the remaining burned gases exit the engine. The transfer function of the fuel tank was found and it was understood that the transfer function is independent from the height of the fluid inside the tank and the area of the partition.

In addition, a PID controller was designed to make the unit step response of the above system has a maximum overshoot of $4.3 \%$ and a peak time of $0.5 \pi$ sec., the steady state error for unit step input to be zero and the dynamic error for ramp input to be $10 \%$. The transfer function of the PID controller was found and the corresponding closed loop transfer function of PID compensated system was calculated. The advantage of PID controller is its feasibility and easy to be implemented. The PID gains can be designed based upon the system parameters if they can be achieved or estimated precisely. Moreover, the PID gain can be designed just based on the system tracking error and treats the system to be "blackbox" if the system parameters are unknown. However, PID controller generally has to balance all threegains impact to the whole system and may compromise the transient response, such as settling time, overshoots, oscillations. ${ }^{[6]}$

\section{Acknowledgment}

We would like to thank Asst. Prof. Melih Çakmakçı, Asst. Prof. Yıldıray Yıldız, Assoc. Prof. Barbaros Çetin, Dr. Hakan Mencek, Münevver Elif Asar, Mert Ali İhsan Kalın, Ömer Benzer, Onuralp Yörük and Xaid Asad for their help during this study.

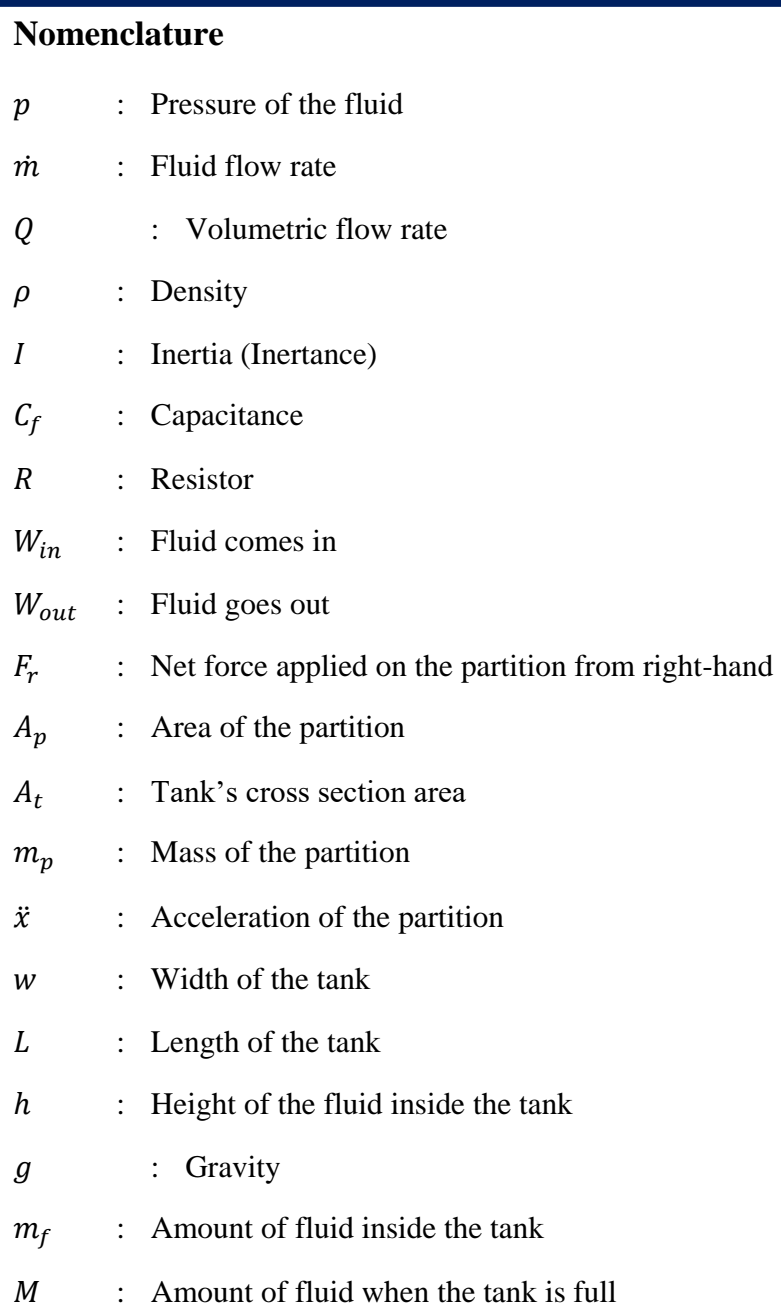

\section{References}

[1] J. Kim. (2007). Fuel Storage Apparatus for Vehicle. U.S Patent, 7231933.

${ }^{[2]}$ Patil, A. and More, S. (2014). Mathematical Modeling of Physical System. IOSR Journal of Electrical and Electronics Engineering, 9, 57-64.

${ }^{[3]}$ Gajic, Z. (2003). Linearization of Nonlinear Systems ,in Linear Dynamic Systems and Signals. Prentice Hall.

[4] "Fuel Tanks and Connectors". Tohatsu Outboards. Retrieved from http://www.tohatsu.com/accessories/fuel_tanks.html

${ }^{[5]}$ Lurie, M. V. (2008). Modeling of Oil Product and Gas Pipeline Transportation. Weinheim, Germany: Wiley-VCH Verlag $\mathrm{GmbH} \& \mathrm{Co} . \mathrm{KGaA}$.

${ }^{[6]}$ Ansorge, R. andSonar, T. (2009). Mathematical Models of Fluid Dynamics: Modeling, Theory, Basic Numerical Facts - 
An Introduction, Weinheim, Germany: Willey-VCH Verlag

GmbH \& Co. KGaA.

[7] "Advantages and Disadvantages of PID Control". Re-

$\begin{array}{lll}\text { searchGate. } & \text { Retrieved }\end{array}$

https://www.researchgate.net/post/Can_you_explain_the_advan

tages_and_disadvantages_of_PID_control_in_PLCs

${ }^{[8]}$ Fackrell, S.; Mastroianni, M. and Rankin, G.W. (2003). Mo-

del of the Filling of an Automotive Fuel Tank. Mathematical

and Computer Modelling - MATH COMPUT MODELLING.

$38,519-532$.

[9] Johnson, T.E. and Kreamer, D.K. (1994). Physical and Mathematical Modeling of Diesel Fuel Liquid and Vapor Movement in Porous Media. Ground Water. 32, 551-560. 